\title{
Cross-immunoreactivity between anti-potato apyrase antibodies and mammalian ATP diphosphohydrolases: potential use of the vegetal protein in experimental schistosomiasis
}

\author{
P Faria-Pinto/****/++, MNL Meirelles*, HL Lenzi**, EM Mota**, MLO Penido***, \\ PMZ Coelho****/*****, EG Vasconcelos/ ${ }^{+}$
}

\begin{abstract}
Departamento de Bioquímica/ICB, Universidade Federal de Juiz de Fora, 36015-400 Juiz de Fora, MG, Brasil *Departamento de Ultraestrutura e Biologia Celular**Departamento de Patologia, Instituto Oswaldo Cruz-Fiocruz, Rio de Janeiro, RJ, Brasil ***Departamento de Bioquímica e Imunologia/ICB, Universidade Federal de Minas Gerais, Belo Horizonte, MG, Brasil ****Centro de Pesquisas René-Rachou-Fiocruz, Belo Horizonte, Minas Gerais, Brasil *****Sta Casa de Misericórdia de Belo Horizonte, Belo Horizonte, MG, Brasil
\end{abstract}

We have previously showed that Schistosoma mansoni ATP-diphosphohydrolase and Solanum tuberosum potato apyrase share epitopes and the vegetable protein has immunostimulatory properties. Here, it was verified the in situ cross-immunoreactivity between mice NTPDases and anti-potato apyrase antibodies produced in rabbits, using confocal microscopy. Liver samples were taken from Swiss Webster mouse 8 weeks after infection with S. mansoni cercariae, and anti-potato apyrase and TRITC-conjugated anti-rabbit IgG antibody were tested on cryostat sections. The results showed that S. mansoni egg ATP diphosphohydrolase isoforms, developed by anti-potato apyrase, are expressed in miracidial and egg structures, and not in granulomatous cells and hepatic structures (hepatocytes, bile ducts, and blood vessels). Therefore, purified potato apyrase when inoculated in rabbit generates polyclonal sera containing anti-apyrase antibodies that are capable of recognizing specifically S. mansoni ATP diphosphohydrolase epitopes, but not proteins from mammalian tissues, suggesting that autoantibodies are not induced during potato apyrase immunization. A phylogenetic tree obtained for the NTPDase family showed that potato apyrase had lower homology with mammalian NTPDases 1-4, 7, and 8. Further analysis of potato apyrase epitopes could implement their potential use in schistosomiasis experimental models.

Key words: cross-immunoreactivity - Schistosoma mansoni ATP diphosphohydrolase - mammalian NTPDases potato apyrase - granuloma

Recently, two actives ATP diphosphohydrolase isoforms were partially purified from Schistosoma mansoni egg (Faria-Pinto et al. 2004). These enzymes, previously detected on the external surface of schistosomula and worm adult tegument (Vasconcelos et al. 1996, De Marco et al. 2003), hydrolyze di- and triphosphate nucleosides and belong to the nucleoside triphosphate diphosphohydrolases (NTPDases) family that share five apyrase-conserved regions (Handa \& Guidotti 1996, Vasconcelos et al. 1996). Potato apyrase was one of the first proteins of this family to be purified (Traverso-Cori et al. 1965). Since then, a number of ATP diphosphohydrolases differing in their catalytic properties and subcellular localization have been identified from different species, such as parasites, mammals and plants, and play important roles in the control of nucleotides levels in a variety of physiological processes (Coimbra et al. 2002, Gendron et al. 2002, Ivanenkov et al. 2005).

Studies in our laboratory showed that potato apyrase purified from Solanum tuberosum presents remarkable im-

Financial support: Fapemig, CNPq

+ Corresponding author: eveline@icb.ufjf.br

${ }^{++}$Fellowship CPqRR

Received 25 May 2006

Accepted 26 June 2006 munostimulatory properties, activating humoral immune responses in mice and rabbits (Vasconcelos et al. 1996, Faria-Pinto et al. 2004) and cellular immune responses in mice, inducing a mixed Th1/Th2-type cytokine secretion profile (unpublished data). Rabbit policlonal antibodies against purified potato apyrase showed strong cross-immunoreactivity with partially purified $S$. mansoni ATP diphosphydrolase isoforms, suggesting that the parasite and vegetable proteins share conserved epitopes (Vasconcelos et al. 1996, Faria-Pinto et al. 2004). In situ confocal fluorescence microscopy demonstrated cross-immunoreactivity between rabbit raised potato apyrase antibodies and S. mansoni ATP diphosphohydrolase on the external surface of miracidium, probably associated with its membrane, in the egg von Lichtenberg's envelope and in the outer side of the eggshell, entrapped by the surface microspines, suggesting that a soluble ATP diphosphohydrolase isoform is secreted (Faria-Pinto et al. 2004). Furthermore, during the acute stage of Swiss Webster schistosomiasis, using potato apyrase as antigen, high levels of Ig specific anti-ATP diphosphohydrolase serum isotypes were observed in ELISA technique, and a Th2 type cytokine secretion profile was notice in vitro, in response to splenic lymphocytes stimulation. These events suggest that this parasite protein could be involved in the host immune response during the course of the schistosome infection (Jacinto et al. 2001, FariaPinto et al. 2004). 
Here, the reactivity of the policlonal rabbit anti-potato apyrase against mice NTPDases was analyzed by laser confocal microscopy, using liver specimens from mouse infected with $S$. mansoni. In addition, distance estimation of the amino acid sequences of several NTPDases was performed and the resultant phylogenetic tree is also shown.

\section{MATERIALS AND METHODS}

Potato apyrase purification and antibodies production in rabbit - Potato apyrase $(52 \mathrm{kDa})$ was purified from a commercial strain of $S$. tuberosum as described by Kettlun et al. (1992). Polyclonal antiserum against potato apyrase was developed in a New Zealand White rabbit that was immunized with three injections of $100 \mu \mathrm{g}$ of the purified potato apyrase in intervals of 15 days, the first being emulsified in Freund's complete adjuvant and the others, in incomplete Freund's adjuvant. Blood was collected after the third immunization and serum was kept to $-20^{\circ} \mathrm{C}$

Animals and immunocytochemistry - Eight-week-old male Swiss Webster mice were subcutaneously infected with 150 cercariae of Belo Horizonte $S$. mansoni isolate obtained from infected Biomphalaria glabrata snails, as described by Smithers and Terry (1965). Liver specimens were taken 8 weeks after infection and frozen in TissueTek OCT Compound by immersion in liquid nitrogen. Cryostat sections (10 $\mu \mathrm{m}$ thick), after adherence on glass slides previously coated with poly-L-lysine, were fixed in acetone for $30 \mathrm{~min}$ at $-20^{\circ} \mathrm{C}$. After drying, the sections were treated by blockade solution, consisting of PBS with $2 \%$ skim-milk, $2.5 \%$ bovine serum albumin plus $8 \%$ calf fetal serum for $45 \mathrm{~min}$ at room temperature and washed 3 times in PBS. The sections were then incubated overnight at $4^{\circ} \mathrm{C}$ in anti-potato apyrase (sera diluted 1:250 in PBS). The slides were washed twice for 15 min each with gentle agitation in PBS and incubated for $60 \mathrm{~min}$ at $37^{\circ} \mathrm{C}$ with TRITC-conjugated donkey anti-rabbit IgG antibody, diluted 1:800 in PBS. The slides were again washed as described above before addition of 1,4-diazabi-cyclo $[2,2,2]$ octane (DABCO). As a control, sections were incubated with secondary antibody alone. To reduce and discriminate the excessive red autofluorescence from the eggshell, sections were analyzed in an inverted confocal laser scanning microscope (LSM 410-Zeiss), applying a narrow long pass filter (LP570) for the green emissions.

Phylogenetic analysis of amino acid sequences of selected NTPDases - The percentage of amino acid identity of the potato apyrase with selected NTPDases, found in National Center for Biotechnology Information (NCBI) database (www.ncbi.nlm.nih.gov), was determined by pairwise alignment using ALIGNp (http://www. infobiogen.fr/services/analyseq/cgi-bin/alignp_in.pl). Distance estimation of amino acid sequences was then performed and a phylogenetic tree was obtained using GeneBee (http://www.genebee.msu.su/).

\section{RESULTS}

To verify whether antibodies produced against potato apyrase would cross-react with mammalian NTPDases, the in situ expression of the enzyme was developed by indirect immunofluorescence, in cryostat liver sections presenting exudative-productive granulomas, applying confocal laser microscopy. The results showed that the antibody anti-apyrase reacted only with miracidial and egg structures, and not with granulomatous cells (Fig. 1). In fact, in the surrounding granulomatous tissues, rich in inflammatory cells such as macrophages, eosinophils, neutrophils, lymphocytes, mast cells and fibroblastic-like cells (Lenzi et al. 1998), the reactivity with potato apyrase failed to give a significant signal, indicating no crossimmunoreactivity with them. Negative reactivity was also

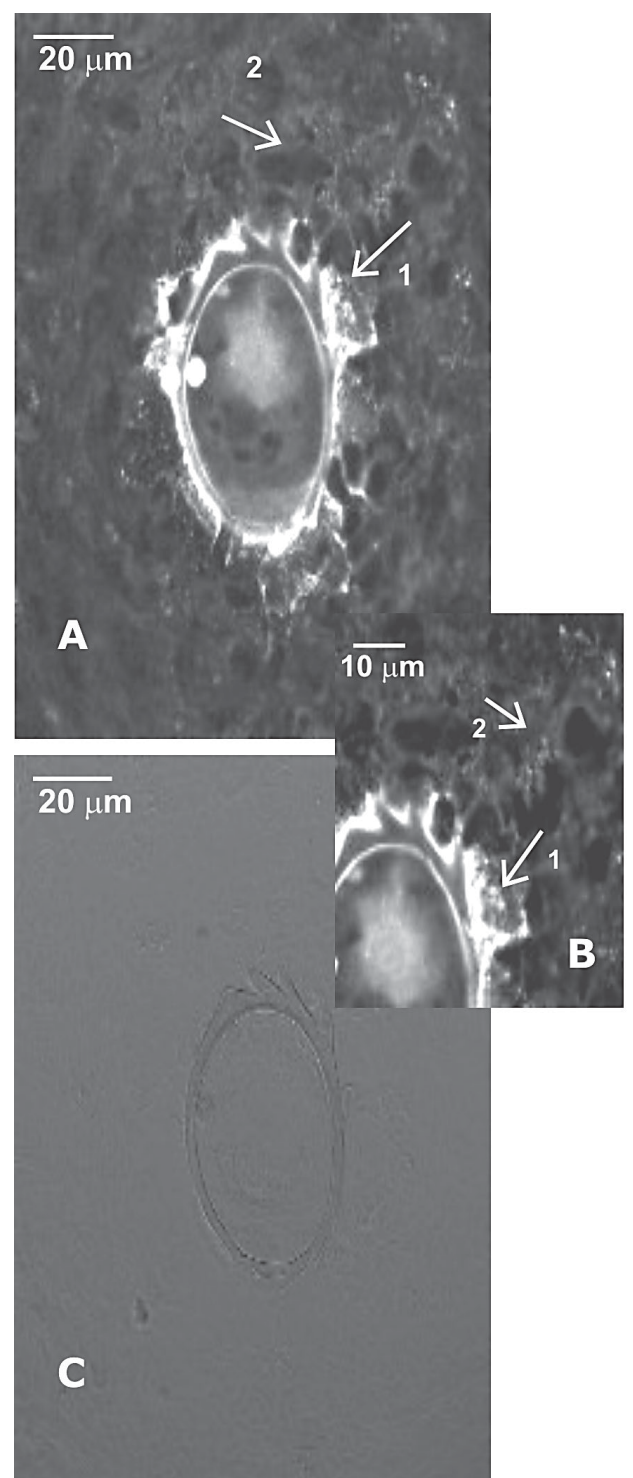

Fig. 1: immunocytochemical localization of the ATP diphosphohydrolase from Schistosoma mansoni egg developed by anti-potato apyrase antibodies and secondary antibody coupled to TRITC, in cryostat sections $(10 \mu \mathrm{m}$ thick) of infected mouse liver and analyzed by laser confocal microscopy. A: general aspect of $S$. mansoni egg showing fluorescence linearly distributed on external surface of miracidium and immediately outside of the egg-shell (arrow 1) and negative reaction in surrounding exudative granuloma structure (arrow 2); B: detail of the image (A); C: DIC image showing tissue integrity and aspect of the egg-shell. 
observed to hepatic parenchyma (Fig. 2A, arrow 1), blood vessels (Fig. 2A, arrow 2), and bile ducts (Fig. 2A, arrow $3)$. No reactivity was also noted with the secondary antibody alone (Fig. 2B). Due to the results obtained by us, the amino acid sequences alignment of potato apyrase with 20 members of the NTPDase family and related proteins were generated using NCBI database. Distance estimation of the amino acid sequences was then performed and the phylogenetic tree obtained is shown in Fig. 3. Potato apyrase amino acid sequence showed the highest homology (45-47\%) and identity (29-31\%) with NTPDases5 and NTPDase6 from mouse and human, and with $S$. japonicum NTPDase (arrow). The other members of mouse and human NTPDases family, such as NTPDases1-4, 7, and 8 showed $36-45 \%$ homology, with lower identity (22$29 \%$ ). Amino acid sequence comparison of the potato apyrase to $S$. mansoni NTPDase1 (arrow) showed all five apyrase-conserved regions previously described for NTPDases family, but low homology among them. It is interesting to note that the phylogenetic tree obtained for the apyrase family indicates that $S$. japonicum NTPDase is phylogenetically closer to potato apyrase and to mammalian NTPDases 5 and 6 than to the other NTPDases.

\section{DISCUSSION}

Here, we showed that purified potato apyrase when inoculated in rabbit generates polyclonal sera containing anti-apyrase antibodies that are capable of recognize specifically the $S$. mansoni ATP diphosphohydrolase egg epitopes but not NTPDases from mammalian tissues.

NTPDases have been described in hepatocytes, epithelial cells of the bile duct system, vascular cells, platelets, lymphocytes, monocytes, and macrophages cells (Gendron et al. 2002, Bigonnesse et al. 2004, Ivanenkov et al. 2005). Eight members of the mammalian NTPDases family have been identified, including six membrane-bound enzymes (NTPDases 1-4, 7, and 8) and two secreted enzymes (NTPDases 5 and 6) (Gendron et al. 2002, Bigonnesse et al. 2004, Ivanenkov et al. 2005). The phylogenetic tree obtained for the NTPDases family (see Fig. 3) showed that potato apyrase had lower homology with mammalian NTPDases 1-4, 7, and 8. This is in accordance with our results, which showed no-reactivity between potato apyrase antibodies and the mice NTPDases. Although we cannot discard a cross-immunoreactivity between anti-potato apyrase and soluble NTPDases, possibly not accessible under our experimental conditions, our results strong suggest that autoantibodies anti-NTPDases are not induced during potato apyrase experimental immunization.

Recently, we partially purified two egg ATP diphosphohydrolase isoforms, one localized on the external surface of miracidium, and the other soluble and released from S. mansoni egg (Faria-Pinto et al. 2004). It is possible that this soluble isoform corresponds to some $S$. mansoni gene not yet identified or an alternative splicing of the gene recently identified (see Fig. 3), with more similarity to potato apyrase and S. japonicum NTPDase, and maybe the principal responsible by high anti-ATP diphosphohydrolase antibodies levels observed by us in experimental schistosomiasis.
Granuloma modulation induced by antigen is an attractive model for immunization studies in experimental schistosomiasis to test the effect of anti-pathology vaccine (Gustavson et al. 2002). The results showed in this work, added to previously described characteristics of the potato apyrase, such as its cross immunoreactivity to S. mansoni ATP diphosphohydrolase isoforms, immunostimulatory properties, and the large amount of highly purified extraction obtainable from $S$. tuberosum potato by conventional methods, could be considered to imple-
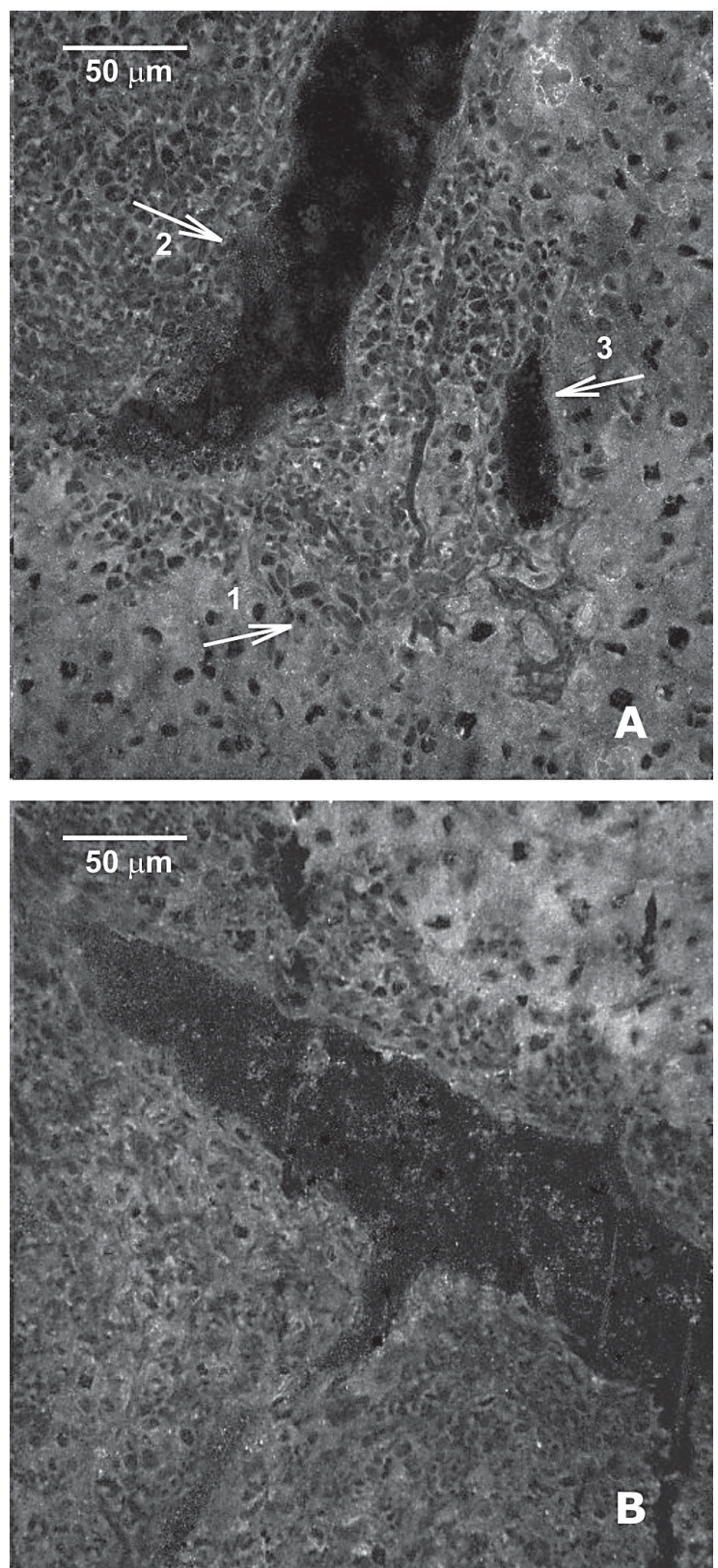

Fig. 2A: mouse hepatocytes (arrow 1), blood vessels (arrow 2) and bile duct (arrow 3), all negatively labeled when probed with antipotato apyrase antibodies and secondary antibody coupled to TRITC used for fluorescence detection of ATP diphosphohydrolase on cryostat sections $(10 \mu \mathrm{m}$ thick) of infected mouse liver, using confocal laser microscopy; B: section probed with secondary antibody alone. 


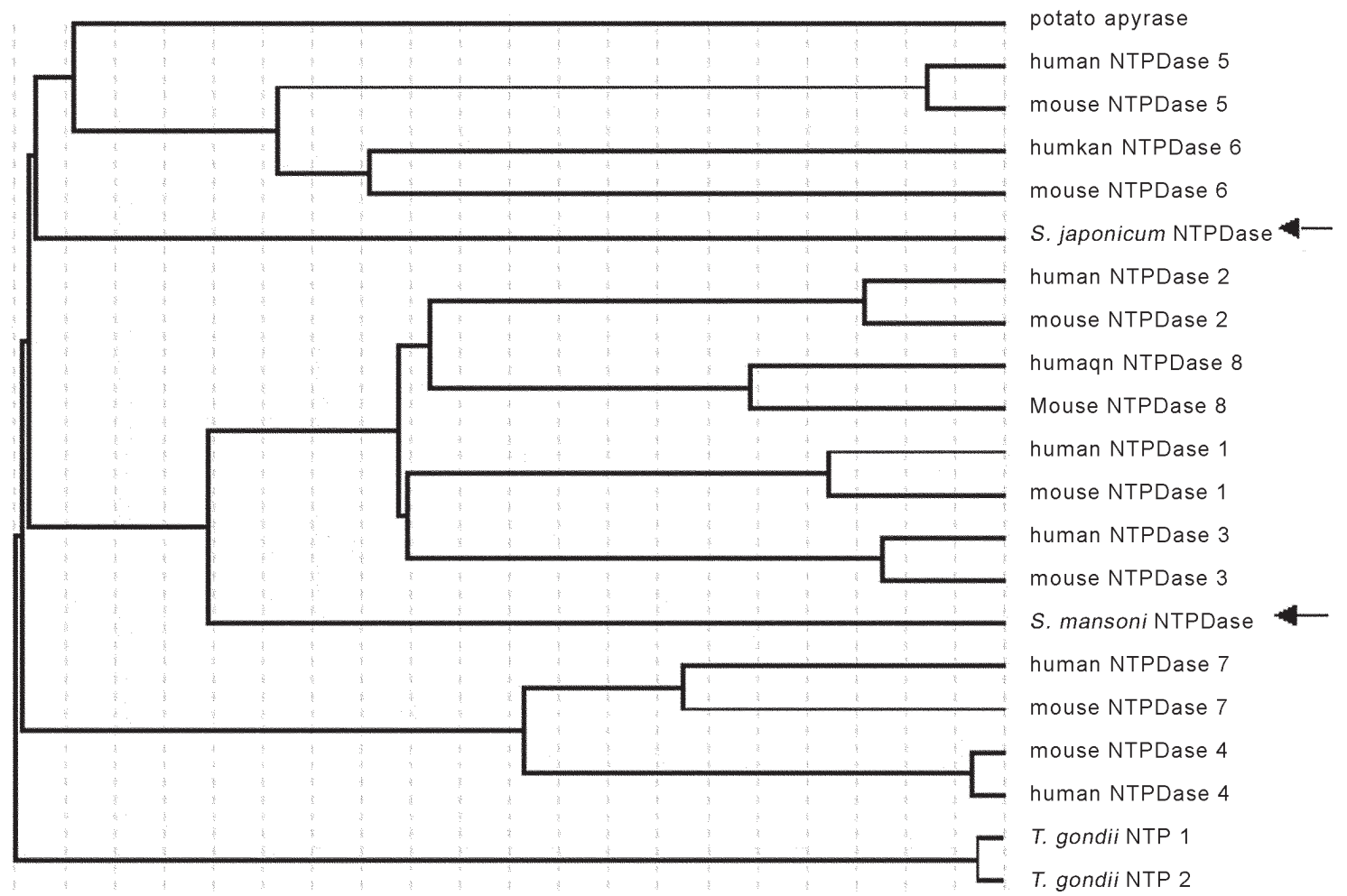

Fig. 3: phylogenetic analysis of amino acid sequences of selected NTPDases. The GeneBank accession numbers of the sequences are as follows: potato apyrase, U58597; mouse NTPDase5, AF006482; human NTPDase5, AF039918; human NTPDase6, AF039916; mouse NTPDase6, AK156685; mouse NTPDase1, AF037366; human NTPDase1, S73813; human NTPDase2, AF144748; mouse NTPDase2, AF042811; mouse NTPDase8, AY364442; human NTPDase8, AY903954; mouse NTPDase3, AK046218; human NTPDase3, AF034840; mouse NTPDase4, AK004761; human NTPDase4, AF016032; human NTPDase7, AF269255; mouse NTPDase7, AF042811; Schistosoma japonicum NTPDase, AY814499; S. mansoni NTPDase, AY323529; Toxoplasma gondii NTP1, U96965; T. gondii NTP2, Q27895.

ment the use of this vegetal protein in experimental immunology. Since potato apyrase is not a highly glycosylated protein (Handa \& Guidotti 1996), we postulated the use of its native form or its derivative antigenic peptides in studies of protective immunogenicity and/or immunotherapy in experimental schistosomiasis.

\section{ACKNOWLEDGEMENTS}

To Dr Jair AK Aguiar for revising the manuscript.

\section{REFERENCES}

Bigonnesse F, Levesque AS, Kukulski F, Lecka J, Robson SC, Fernandes MJG, Sevigny J 2004. Cloning and characterization of mouse nucleoside triphosphate diphosphohydrolase8. Biochemistry 43: 5511-5519.

Coimbra ES, Gonçalves Da Costa SC, Corte-Real S, Freitas FGR, Durão A, Souza CSF, Silva Santos MI, Vasconcelos EG 2002. Characterization and cytochemical localization of an ATP diphosphohydrolase from Leishmania amazonensis promastigote. Parasitology 124: 137-143.

DeMarco R, Kowaltowski AT, Mortara RA, Verjovski-Almeida S 2003. Molecular and characterization of Schistosoma mansoni ATP diphosphohydrolase. Biochem Biophys Res Commun 307: 831-838.

Faria-Pinto P, Meirelles MNL, Penido MLO, Lenzi HL, Coelho
PMZ, Vasconcelos EG 2004. ATP diphosphohydrolase from Schistosoma mansoni egg: characterization of a new antigen. Parasitology 129: 51-57.

Gendron FP, Benrezzak O, Krugh BW, Kong Q, Weisman GA, Beaudoin AR 2002. Purine signaling and potential new therapeutic approach: possible outcomes of NTPDase inhibition. Current Drug Targets 3: 229-245.

Gustavson S, Zouain CS, Alves JB, Leite MF, Goes AM 2002. Modulation of granulomatous hypersensitivity against Schistosoma mansoni eggs in mice vaccinated with culture-derived macrophages loaded with PIII. Parasitol Int 51: 259269.

Handa M, Guidotti G 1996. Purification and cloning of a soluble ATP diphosphohydrolase (apyrase) from potato tubers (Solanum tuberosum). Biochem Biophys Res Commun 218: 916-923.

Ivanenkov VV, Meller J, Kirley TL 2005. Characterization of disulfide bonds in human nucleoside triphosphate diphosphohydrolase 3 (NTPDase3): implications for NTPDase structural modeling. Biochemistry 44: 8998-9012.

Jacinto AA, Bordin FHS, Souza EE, Ferreira AP, Montesano MA, Teixeira HC, Penido MLO, Coelho PMZ, Vasconcelos EG 2001. Análise de anticorpos e citocinas produzidas em resposta a ATP difosfohidrolase de Schistosoma mansoni 
durante o estágio agudo da esquistossomose experimental. Principia 6: 83-92.

Ketllun AM, Urra R, Leyton M, Valenzuela MA, Mancilla M, Traverso-Cori A 1992. Purification and characterization of two isoapyrases from Solanum tuberosum var. ultimus. Phytochemistry 31: 3691-3696.

Lenzi HL, Kimmel E, Schechtman H, Pelajo-Machado M, Romanha WS, Pacheco R, Mariano M, Lenzi JA 1998. Histoarchitecture of schistosomal granuloma development and involution: morphogenetic and biomechanical approaches. Mem Inst Oswaldo Cruz 93: 141-151.
Smithers SR, Terry RJ 1965. The infection of laboratory hosts with cercariae of Schistosoma mansoni and the recovery of adult worms. Parasitology 55: 695-700.

Traverso-Cori A, Chaimovich H, Cori O 1965. Different molecular forms of potato apyrase. Arch Biochem Biophys 137: 133-142.

Vasconcelos EG, Ferreira ST, Carvalho TMU, Souza W, Kettlun AM, Mancilla M, Valenzuela MA, Verjovski-Almeida S 1996. Partial purification and immunohistochemical localization of ATP diphosphohydrolase from Schistosoma mansoni. J Biol Chem 271: 22139-22145. 
